Results The application was developed in the Engine Unity that allows platform portability and friendly interactions from the definition of visual contexts aligned with the target audience, adolescents and young Catholics. The contents selected to be placed in the mobile application were selected from the contents of the Adolescent Chat site, and focus on the themes of Sexuality, STD/HIV/AIDS and STD Prevention. The contents were developed and validated in a previous study. In addition to the presentation of explanatory content, the adolescent has the option of interacting through illustrations, animations and quizzes.

Conclusion The relevance of this study is therefore acknowledged, since it is focused on the development of an educational technology in health in the virtual format, aimed at adolescents, using recreational methods that favour critical reflection and, consequently, adoption of safe sexual behaviours. The importance of the application as an instrument of health education is highlighted, enhancing the actions of STD/ HIV/AIDS prevention, solidifying the interdisciplinary practice, mainly aimed at improving the care of adolescents.

\section{P4.82 MOBILE APPLICATIONS FOR ADOLESCENTS AVAILABLE ON VIRTUAL PLATFORMS: HEALTH PROMOTION POSSIBILITIES} ${ }^{2}$ Adriana Gomes Nogueira Ferreira, ${ }^{3}$ Luisa Fânia da Costa Pinheiro. ' Universidade Federal do Ceará, Fortaleza - CE, Brazil; ${ }^{2}$ Universidade Federal do Maranhão, Imperatriz - MA, Brazil; ${ }^{3}$ Universidade Estadual do Ceará, Fortaleza - CE, Brazil

\subsection{6/sextrans-2017-053264.577}

Introduction Children and adolescents constitute population connected to screens and digital technologies. They invest in the interactive process, periods of time that would be available for leisure, entertainment, study, school tasks, social and family relationships and other daily activities. In this context, this study aims to relate the mobile applications on sexuality and STD/HIV/AIDS prevention available on the main existing platforms.

Methods The list of applications was based on the Google Play (Android), App Store (iOS) and Marketplace (Windows Phone) search terms: Adolescent/teenager, sexuality, isolated and associated STD/HIV prevention, on both platforms, in the period between March and April 2016. Subsequently, the five most popular applications were identified from the identification of the available note and the number of evaluators. To analyse the data the main characteristics of the applications aimed at adolescents and discussed with the relevant literature were identified. The study obeyed the ethical aspects related to research involving human beings.

Results The research revealed that the most popular applications were games and erotic content, it is noted that no applications were found that contemplated adolescence and disease prevention. The most popular applications had scores ranging from 4.0 to 4.7 from a maximum score of 5.0 points. Of the five most popular, three belonged to the Android operational system and two to the Windows Phone operational system, of these, two propose to follow menstrual cycle, thus contemplating aspects of health promotion, and the others were games.

Conclusion The increasing use of information and communication technologies in the health area raises the need to include the concept of health care promotion using features such as mobile applications developed for smartphones and tablets.
Therefore, the importance of adapting the contents of mobile devices is extended, extending its use to information and health education.

\section{P4.83 HIGH RISK BEHAVIOUR AND DETERMINANTS OF PATRONAGE OF HIV COUNSELLING AND TESTING AMONG BROTHEL-BASED FEMALE SEX WORKERS IN LAGOS, NIGERIA}

${ }^{1}$ Peter Adeyeye, ${ }^{2}$ Mohammed Bello, 'Shola Gbadamosi. 'University of Lagos, Lagos, Nigeria; ${ }^{2}$ Centre for Research and Documentation, Kano, Nigeria

\subsection{6/sextrans-2017-053264.578}

Introduction Sex workers are heightened risk of HIV/AIDS because of their risky sexual behaviour. In Nigeria, the number of sex workers living with HIV is eight times higher than the general population. HIV counselling and testing (HCT) is important in reducing spread of HIV and serves as an entry point to care. The study aimed at understanding the factors that influences sex workers patronage of HCT services in the study area.

Methods The study was conducted in October 2016 among 150 brothel-based Female Sex Workers (FSWs) purposively selected across three randomly selected local government areas representing the three senatorial districts in Lagos, Nigeria. The result was processed through SPSS 16.0. A binary logistic regression model was used in understanding the predictors of HIV testing among the sex workers.

Results The mean age of sex workers was 28 years. Findings showed a high prevalence of risky behaviour with about twothird (65.3\%) consumed alcohol and 42\% sometimes engaged in unprotected sex. Majority (86\%) had gone for HIV testing prior to the survey but only seven in every ten went for HCT within six months prior to the study. Results from the binary logistic regression showed that sex workers education and length of practicing sex work exert a statistical significance on the patronage of HIV testing $(\mathrm{p}<0.05)$. Sex workers that do not engage in unprotected sex are less likely to patronise HCT (OR 0.53) than those that sometimes have unprotected sex while sex workers that are not aware of HCT centres are less likely (OR 0.85) to patronise HCT services.

Conclusion Findings demonstrate high risky sexual behaviour that exposes the FSWs to HIV infection. While a significant number of the sex workers have accessed HCT services at some point, many did not patronise within six months to the study. Though many attested to regular visit of NGOs involved in HCT testing in their brothels, there is still need for these NGOs to work with brothel managers. There is also an imperative for sexuality education to reduce high risky behaviour such as unprotected sex.

\section{P4.84 HOW MANY ACCOUNT FOR HOW MUCH? LORENZ CURVES AND THE CONCENTRATION OF CONDOMLESS SEX PARTNERSHIPS}

${ }^{1}$ Peter Saxton, ${ }^{1}$ Andrew Pasley, ${ }^{2}$ Anthony Hughes, ${ }^{3}$ Nigel Dickson, ${ }^{2}$ Adrian Ludlam. ${ }^{1}$ University of Auckland, Auckland, New Zealand; ${ }^{2}$ New Zealand AIDS Foundation, Auckland, New Zealand; ${ }^{3}$ University of Otago, Dunedin, New Zealand

10.1136/sextrans-2017-053264.579 